\title{
Using computers for continuing professional development in otolaryngology
}

As clinicians, we are expected to continue our learning and professional development throughout our working lives. Doctors have a duty to 'keep their professional knowledge and skills up to date ${ }^{1}$ and to acquire new skills for the benefit of their patients. Continuing professional development (CPD) is the process by which individual doctors keep themselves up to date in order to maintain high standards of professional practice, ${ }^{2}$ to improve their clinical competence and patient care, to comply with accepted standards, and to contribute towards clinical governance and revalidation, as well as to help progress their careers. ${ }^{3}$

Various activities can constitute CPD, including: attendance or presentations at meetings, conferences and courses; teaching and examinations and self-directed learning such as reading journals, information searches, undertaking research and contributing to the published literature. ${ }^{4}$ Much of this is resource-intensive - and particularly timeintensive - and, with increasingly busy lives, doctors often find it difficult to maintain a reasonable work-life balance.

Computers are continuing to play a larger part in the CPD process as they enable access to information 24 hours a day. Modern technology offers smaller and increasingly more powerful mobile access to such information. There is also no doubt that the advent of multimedia applications has enriched the learning experience and improved the learning process.

In this article, some of the applications and resources that are currently available to enhance clinician learning and professional development are introduced. Further details of these applications and links to relevant websites can be found at The Journal of Laryngology \& Otology website (www. jlo.co.uk).

\section{Personal digital assistant resources}

\section{AvantGo content delivery service}

Web browsers for personal digital assistants (PDAs) have come a long way recently but still do not offer the most usable way of viewing web pages that were originally designed for personal computer (PC) monitors. AvantGo ${ }^{\circledR}$ (www.AvantGo.com) is a service which packages web content for handheld computer access and which enables browsing of content offline. This application allows the user the opportunity either to create customised channels for their PDA from any website or to subscribe to one of the many freely available channels, including those carrying news and sport as well as information on health-related matters. Once registered, it is possible to download software that allows viewing of material on a PDA; it is also easy to synchronise and to rapidly update this material. The advantage of such an application is that content from various resources can be collected in one location and updates can easily be synchronised via a desktop $\mathrm{PC}$ internet connection or directly via a wireless connection. Many websites, including those for journals, now offer Really Simple Syndication (RSS) feeds which can also be accessed as an AvantGo channel. Such feeds are essentially a link to information which is updated dynamically and many otolaryngology journals now offer such feeds of current journal abstracts.

The Journal of Laryngology \& Otology offers a version of the current issue as an AvantGo channel in order to enable subscribers to read articles on their PDA. Thus, subscribers can access the full text of all main articles wherever they are, without having to carry the paper edition (Figure 1).

\section{MobiPocket reader}

The MobiPocket (www.mobipocket.com) is a universal reader for personal digital assistants that allows easy reading of e-books. E-medicine (www.emedicine.com) offers an otolaryngology e-textbook that can be downloaded, such that this knowledge base can be accessed directly and easily in any location. The search functionality enables you to find any chosen topic and also to highlight areas of text, add bookmarks and even insert personalised clinical notes.

\section{RepliGo}

RepliGo $^{\mathrm{TM}}$ (www.cerience.com) document mobility software enables viewing of virtually any document via a PDA. It converts Microsoft Office documents, portable document format (PDF) files, web pages and hundreds of other documents into replicas which can then be viewed easily. The advantage of such documents is the ability to zoom and to text wrap to the screen, thus eliminating the need for tedious horizontal scrolling on the PDA. All content (such as fonts, charts, graphics, images and tables) can easily be viewed using a streamlined document navigation interface. This application is also ideal for storing PowerPoint slideshows on a PDA, either for personal perusal or for presentation via a data projector. 


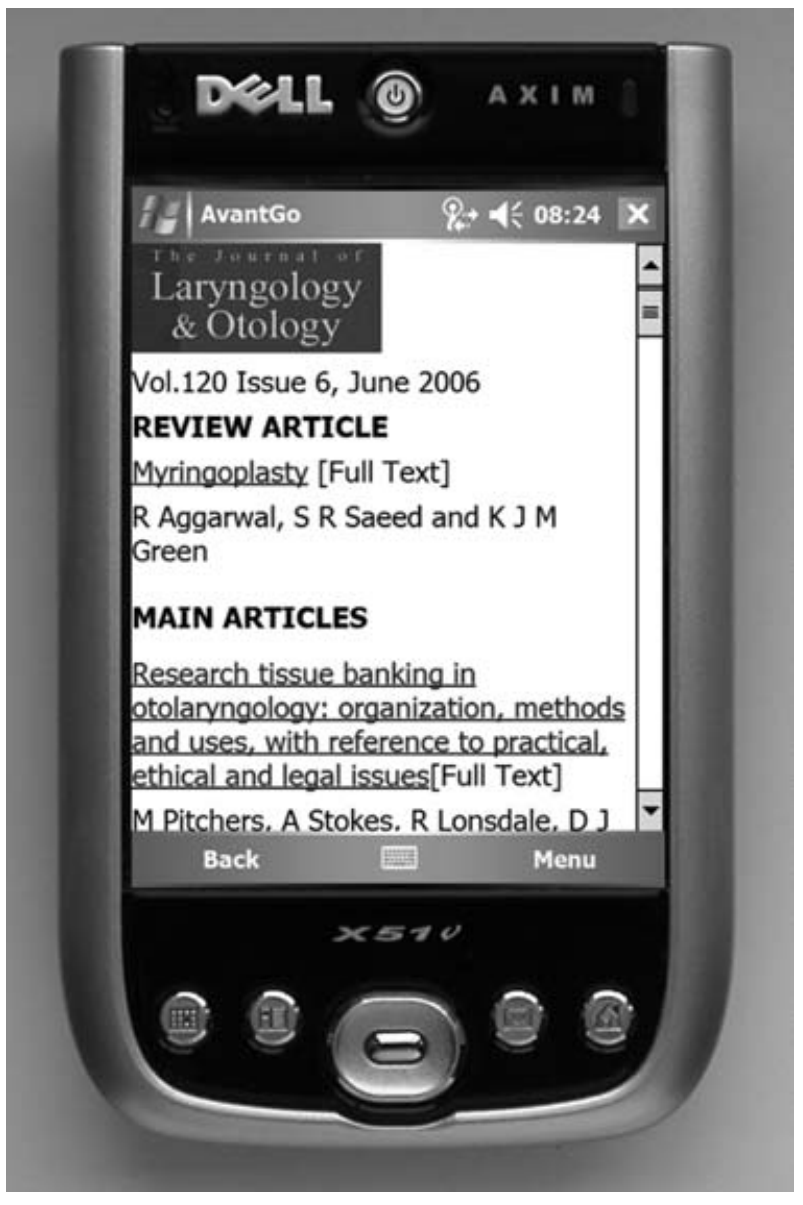

FIG. 1

Personal digital assistant view of The Journal of Laryngology \& Otology content, accessed via AvantGo ${ }^{\circledR}$.

A RepliGo document is created, following software installation, by simply printing the document after selecting the RepliGo software as the printer. This will then print to file and create a RepliGo document at the user's location of choice.

\section{Electronic journals \\ e-Journals}

The internet has changed the way information is received and stored. Only a few years ago, pages of information required a filing cabinet for storage and to enable reference at a later date. Nowadays information is stored electronically and important documents are painlessly located via powerful search functions. Electronic journals are able to provide linking to cross references and to Medline directly, with the added advantages of saving searches and articles for future use. Many journals have utilised this technology and offer an electronic version of their content. For example The Journal of Laryngology \& Otology currently offers free access to abstracts of each issue as well as to back issues to 2000; subscribers can also access full articles in PDF and hypertext markup language (HTML) formats. In the future all previous issues will be available electronically, which will provide a huge educational resource that was previously only accessible through national libraries. This resource will be available worldwide through the internet.

\section{Forthcoming articles}

An exciting development in journal publishing is incremental publishing. This allows work to be published online ahead of its paper publication; it can thus be cited much faster than was previously possible. When an article is published online it receives a unique digital object identification (DOI) number and is thus universally accepted as a published document which can be quoted. Authors benefit from their work being released in a timely fashion and readers benefit from early access to important research findings.

\section{Abstract selection}

Keeping abreast of recent developments in the field involves scan-reading abstracts from many different journals, which requires time. A useful method of receiving a selection of abstracts from nominated journals is provided by Amedeo (www.amedeo. com); selected abstracts are sent directly to subscribers' e-mail in-boxes every week.

\section{Information searches \\ Bioinformatics toolbar}

The MolBiolNet toolbar (www.bioinformatics.vg/ toolbar), added to one's internet browser, is the fastest and easiest way to get information from National Center for Biotechnology Information (NCBI) databases such as PubMed and from other biological web resources. MolBiolNet's normal search engine facility allows searching of Google and the Amazon books directory and contains links for fast redirection to literature databases. The MolBiolNet Toolbar is free to download and appears every time a new Internet Explorer window is opened.

\section{Google Scholar}

Google Scholar (www.scholar.google.com) provides a very simple way to search the literature. From one site many sources can be searched, including peer-reviewed papers, theses, books, abstracts and articles. In ranking the article, Google takes into account the text, the author, the journal and the number of times the article has been cited.

\section{Google Alerts}

With rapid changes in the processes of healthcare delivery, patients are often more aware than clinicians of new medical developments. Google Alerts (www.google.com/alerts) offers to deliver into subscribers' e-mail in-boxes the results of their chosen searches from various news and web resources, thus ensuring that they do not miss important medical news articles. 


\section{PubMed alerts}

PubMed (www.pubmed.gov) is a service of the US National Library of Medicine which includes over 16 million citations from Medline and from other life science journals as well as other biomedical articles. It searches back to the 1950s. PubMed offers updated e-mail alerts of subscribers' chosen information searches as and when new articles appear in the literature. Subscribers can choose to receive these alerts weekly, monthly or as often as requested. For example you can be alerted when the next article is published on 'stapedectomy' and you are then supplied with a link to the full abstract.

\section{Newsreaders}

With more than a billion web pages available, it can be somewhat time-consuming to find the information one needs by clicking from site to site. Really Simple Syndication feeds alert subscribers when nominated sites (from throughout the internet) have added new content. The latest headlines and articles (and even audio files, photographs and videos) can be accessed in one place, as soon as they are published, without the user having to remember to visit each site regularly. Really Simple Syndication feeds are a type of web page designed to be read by a Newsreader application. Many websites, including many otolaryngology journal websites, now offer RSS feeds of their content allowing one to effortlessly receive abstracts of current journal issues at one location. There are many Newsreader applications available, including Google Reader (www.google.com/reader).

\section{Electronic resources}

There are many useful electronic resources available including on-line web resources and off-line digital video disk (DVD) multimedia resources. Such is the variety that there are really too many to be listed within the confines of this article. However, they include: online specialist libraries for ENT and audiology; resources for clinical guidance and evidence-based medicine, clinical databases and electronic books and journals. Various practical guides to surgery are also becoming available, with interactive DVDs that incorporate text, images, sound and video. More details and links can be found by visiting The Journal of Laryngology \& Otology website (www.jlo.co.uk).

\section{The future}

With the capability to view simultaneous text, images, sound and video via computer, the e-learning experience has been enhanced and enriched. The future holds the promise of increased e-learning opportunities for clinicians with the advent of multimedia resources approved for CPD available through the medical royal colleges. ${ }^{5}$

In common with other journals, The Journal of Laryngology \& Otology has been working on providing such content, and it hopes to start in the near future by providing multimedia presentations of lectures given by its visiting professors. These presentations will allow online streaming of the lecture slides, together with a video of the presenter, so that a virtual experience akin to being in the lecture theatre is provided.

The future is exciting, and we at The Journal of Laryngology \& Otology hope to be a part of it by providing, through the website, a range of materials that we hope will act both as adjuncts to the written word and also as educational resources for our subscribers.

\section{References}

1 The duties of a doctor. General Medical Council (UK). http://www.gmc-uk.org [25 July 2006]

2 Ten principles for continuing professional development. Academy of Medical Royal Colleges (UK). http://www. aomrc.org.uk/ [25 July 2006]

3 Continuing professional development, a manual for SIGN guideline developers. Scottish Intercollegiate Guidelines Network. http://www.sign.ac.uk [25 July 2006]

4 Continuing professional development for UK Physicians. Federation of the Royal College of Physicians of UK http://www.rcplondon.ac.uk/ [25 July 2006]

5 Executive summary of the meeting of the academy of medical royal colleges (UK). Academy of Medical Royal Colleges (UK). http://www.aomrc.org.uk/ [25 July 2006] 